\title{
Dark energy and dark matter from hidden symmetry of gravity model with a non-Riemannian volume form
}

\author{
Eduardo Guendelman $^{1, \mathrm{a}}$, Emil Nissimov ${ }^{2, \mathrm{~b}}$, Svetlana Pacheva ${ }^{2, \mathrm{c}}$ \\ ${ }^{1}$ Department of Physics, Ben-Gurion University of the Negev, Beersheba, Israel \\ ${ }^{2}$ Institute for Nuclear Research and Nuclear Energy, Bulgarian Academy of Sciences, Sofia, Bulgaria
}

Received: 13 August 2015 / Accepted: 22 September 2015 / Published online: 3 October 2015

(C) The Author(s) 2015. This article is published with open access at Springerlink.com

\begin{abstract}
We show that dark energy and dark matter can be described simultaneously by ordinary Einstein gravity interacting with a single scalar field provided the scalar field Lagrangian couples in a symmetric fashion to two different spacetime volume forms (covariant integration measure densities) on the spacetime manifold - one standard Riemannian given by $\sqrt{-g}$ (square root of the determinant of the pertinent Riemannian metric) and another non-Riemannian volume form independent of the Riemannian metric, defined in terms of an auxiliary antisymmetric tensor gauge field of maximal rank. Integration of the equations of motion of the latter auxiliary gauge field produce an a priori arbitrary integration constant that plays the role of a dynamically generated cosmological constant or dark energy. Moreover, the above modified scalar field action turns out to possess a hidden Noether symmetry whose associated conserved current describes a pressureless "dust" fluid which we can identify with the dark matter completely decoupled from the dark energy. The form of both the dark energy and dark matter that results from the above class of models is insensitive to the specific form of the scalar field Lagrangian. By adding an appropriate perturbation, which breaks the above hidden symmetry and along with this couples dark matter and dark energy, we also suggest a way to obtain growing dark energy in the present universe's epoch without evolution pathologies.
\end{abstract}

\section{Introduction}

The late time Universe appears to be dominated by two components, both of them "non-luminous" or "dark". The dominant contribution, about $70 \%$ of the energy density of the

\footnotetext{
a e-mail: guendel@bgu.ac.il

be-mail: nissimov@inrne.bas.bg

c e-mail: svetlana@inrne.bas.bg
}

universe is well described by a cosmological constant term, as introduced originally by Einstein and has also been given the name "dark energy". This contribution leads to gravitational repulsion. The cosmological constant or dark energy is not diluted by the expansion of the universe. The other subdominant contribution, about $25 \%$ of the energy density of the universe is well described by a pressureless fluid, which is called "dark matter". As opposed to the dark energy it is gravitationally attractive and it gets diluted by the universe expansion, it can form structures, etc.

Dark energy was observationally discovered rather recently through the observation of type Ia supernova [1-3].

Dark matter was first postulated in the 1930s, separately by Oort and Zwicky, due to the anomaly of the orbital velocity of some stars in the Milky Way galaxy and the orbital velocity of galaxies in clusters. A recent review of dark matter is given in Ref. [4], reviews of dark energy can be found in $[5,6]$ and a review of both dark matter and dark energy in [7].

In this paper we study a class of models providing a unified description of dark energy and dark matter starting from a well-defined gravity-scalar-field Lagrangian action constructed by means of both standard Riemannian as well as an alternative non-Riemannian (i.e., independent of the pertinent Riemannian metric) volume forms (covariant integration measure densities) on the spacetime manifold. The introduction of such a modified "two-measure" gravity-matter theory (the general class of "two-measure" gravity models was originally proposed in Refs. [8-11]) opens the possibility to obtain both dark energy and dark matter from a single scalar field, as was already observed in [12]. This was further generalized in [13] by the inclusion of another field with a phantom-like kinetic energy so as to produce growing dark energy. In the present paper we will achieve growing dark energy in a different way, which does not invoke phantom kinetic terms and without introducing additional fields.

In a recent paper [14] a model providing unifying description of dark energy and dark matter was proposed by studying 
thermodynamics of cosmological systems where a constraint on the pressure being a constant was introduced from the very beginning. In the present case we start from a well-defined Lagrangian action principle for a modified gravity-scalarfield system which produces systematically the constant pressure constraint in a self-consistent dynamical way as part of the pertinent equations of motion.

Here we will proceed to discover the fundamental reasons of how modified gravity-matter models, generalizing those studied in Ref. [12], succeed to describe simultaneously both dark matter and dark energy. We find that this is realized due to:

- (i) The existence of a hidden (strongly nonlinear) Noether symmetry of the underlying single scalar field Lagrangian, which implies a conservation law from which it follows that there is conserved current giving rise to the dark matter component.

- (ii) An a priori arbitrary integration constant appears in a dynamical constraint on the scalar field Lagrangian, which plays the role of a dynamically generated cosmological constant and provides the dark energy component. The fact that the latter arises from an integration constant makes the observed vacuum energy density totally decoupled from the parameters of the matter Lagrangian.

The two fundamental features (i)-(ii) arise in a way completely independent of the specific form of the scalar field Lagrangian and the details of the scalar field dynamics.

Other treatments that unify dark energy and dark matter have appeared before, for example, the Chaplygin gas models [15-18].

More recently, a "mimetic" dark matter model was proposed $[19,20]$ based on a special covariant isolation of the conformal degree of freedom in Einstein gravity, whose dynamics mimics cold dark matter as a pressureless "dust". Also, the cosmological implications of the "mimetic" matter were studied in some detail (second Refs. [19,20]). For further generalizations and extensions of "mimetic" gravity, see Ref. [21].

Models of explicitly coupled dark matter and dark energy described in terms of two different scalar fields were proposed in Ref. [22].

As a final introductory remark let us briefly describe the usefulness of employing the formalism based on alternative non-Riemannian spacetime volume forms, i.e., alternative covariant integration measure densities in gravity-matter Lagrangian actions independent of the pertinent Riemannian metric. The latter have profound impact in any field theory models with general coordinate reparametrization invariance - general relativity and its extensions, strings and (higher- dimensional) membranes as already studied in a series of previous papers on this subject [8-11,23-26].

Although formally appearing as (almost) "pure-gauge" dynamical degrees of freedom ${ }^{1}$ the non-Riemannian volumeform fields trigger a number of remarkable physically important phenomena:

- A non-Riemannian volume-form formalism in gravitymatter theories naturally generates a dynamical cosmological constant as an arbitrary dimensionful integration constant. At this point it resembles the earlier proposed unimodular gravity formulated as a fully generally covariant theory within the framework of Dirac's constraint Hamiltonian method [29]. ${ }^{2}$ Unimodular gravity became further an object of active studies - for the latest developments, especially path integral quantization, equivalence with the fully diffeomorphism invariant formulation, and further references, see [34]. On the other hand, the non-Riemannian volume-form approach goes well beyond the dynamical cosmological constant generation and has significantly broader scope. Namely, unimodular gravity in its generally covariant form (Eq. (18) in [29], which appears as a particular case of a gravity theory with a non-Riemannian volume form) is equivalent to standard general relativity (on classical level, except that the cosmological constant is an integration constant). On the other hand, generic non-Riemannian-volume-form-modified gravity theories are non-trivial extensions to general relativity; see also the points below.

- Employing two different non-Riemannian volume forms generates several independent arbitrary integration constants leading to the construction of a new class of gravity-matter models, which produce an effective scalar potential with two infinitely large flat regions [27,35]. This allows for a unified description of both early universe inflation and of the present dark energy epoch.

- A remarkable feature is the existence of a stable initial phase of non-singular universe creation preceding the inflationary phase - a stable "emergent universe" without "Big-Bang" [35].

- Within non-Riemannian-modified-measure minimal $N=1$ supergravity the dynamically generated cosmological constant triggers spontaneous supersymmetry breaking and mass generation for the gravitino (supersymmetric Brout-Englert-Higgs effect) [36,37]. Applying the same non-Riemannian volume-form formalism to

\footnotetext{
$\overline{1}$ For a detailed canonical Hamiltonian analysis à la Dirac of gravitymatter theories with several independent non-Riemannian spacetime volume forms, see [27] and Appendix A in [28]; see also Sect. 2 for the simple case of one non-Riemannian volume form.

2 The original idea of unimodular gravity is in Einstein's work [30,31]; in a more modern context it appeared in $[32,33]$.
} 
anti-de Sitter supergravity allows one to produce simultaneously a very large physical gravitino mass and a very small positive observable cosmological constant $[36,37]$ in accordance with modern cosmological scenarios for the slowly expanding universe of the present epoch [1-3].

- Adding interaction with a special nonlinear ("squareroot" Maxwell) gauge field (known to describe charge confinement in flat spacetime) produces various phases with different strengths of confinement and/or with deconfinement, as well as gravitational electrovacuum "bags" partially mimicking the properties of MIT bags and solitonic constituent quark models (for details, see [28]).

In Sect. 2 we briefly describe the basics of the nonRiemannian volume-form (modified-measure) approach, including elucidating the meaning of the dynamically generated cosmological constant (i.e., dark energy appearing as an arbitrary integration constant in a dynamical constraint on the scalar field Lagrangian) from the point of view of the canonical Hamiltonian formalism. In Sect. 3 we derive the hidden symmetry and the associated Noether conserved current of the present modified-measure gravity-scalar-field model leading to the "dust-fluid" interpretation of a part of the scalar field energy density, i.e., dark matter. In Sect. 4 few implications for cosmology are considered. We briefly discuss perturbing our modified-measure gravity-scalar-field model which breaks the above crucial hidden symmetry and triggers (upon appropriate choice of the perturbation) a growing dark energy in the present day universe' epoch without invoking any pathologies of "cosmic doomsday" or future singularities kind [38,39], see also [40]. Our concluding remarks are in Sect. 5.

\section{Gravity-matter formalism with a non-riemannian volume-form}

Our starting point is the following non-conventional gravityscalar-field action (for simplicity we use units with the Newton constant $\left.G_{N}=1 / 16 \pi\right)$ :

$S=\int \mathrm{d}^{4} x \sqrt{-g} R+\int \mathrm{d}^{4} x(\sqrt{-g}+\Phi(B)) L(\varphi, X)$,

which in fact is a simple particular case of the general class of the so called "two-measure" gravity-matter theories proposed more than a decay ago [8-11]. The notations we are using are as follows:

- The first term in Eq. (1) is the standard Hilbert-Einstein action; $\sqrt{-g} \equiv \sqrt{-\operatorname{det}\left\|g_{\mu \nu}\right\|}$ is the standard Rieman- nian integration measure density with $g_{\mu \nu}$ being the standard Riemannian spacetime metric.

- $\Phi(B)$ denotes an alternative non-Riemannian generally covariant integration measure density defining an alternative non-Riemannian volume form on the pertinent spacetime manifold:

$\Phi(B)=\frac{1}{3 !} \varepsilon^{\mu \nu \kappa \lambda} \partial_{\mu} B_{\nu \kappa \lambda}$

where $B_{\mu \nu \lambda}$ is an auxiliary maximal rank antisymmetric tensor gauge independent of the Riemannian metric.

$B_{\mu \nu \lambda}$ Eq. (2) will also be called a "measure gauge field". 3

- $L(\varphi, X)$ is a general-coordinate invariant Lagrangian of a single scalar field $\varphi(x)$ of a generic "k-essence" form [42-45]:

$$
\begin{aligned}
L(\varphi, X) & =\sum_{n=1}^{N} A_{n}(\varphi) X^{n}-V(\varphi), \\
X & \equiv-\frac{1}{2} g^{\mu \nu} \partial_{\mu} \varphi \partial_{\nu} \varphi,
\end{aligned}
$$

i.e., a nonlinear (in general) function of the scalar kinetic term $X$.

Varying Eq. (1) w.r.t. $g^{\mu \nu}, \varphi$, and $B_{\mu \nu \lambda}$ yields the following equations of motion, respectively:

$R_{\mu \nu}-\frac{1}{2} g_{\mu \nu} R=\frac{1}{2} T_{\mu \nu}$

$T_{\mu \nu}=g_{\mu \nu} L(\varphi, X)+\left(1+\frac{\Phi(B)}{\sqrt{-g}}\right) \frac{\partial L}{\partial X} \partial_{\mu} \varphi \partial_{\nu} \varphi ;$

$\frac{\partial L}{\partial \varphi}+(\Phi(B)+\sqrt{-g})^{-1} \partial_{\mu}\left[(\Phi(B)+\sqrt{-g}) g^{\mu \nu} \partial_{\nu} \varphi \frac{\partial L}{\partial X}\right]=0 ;$

$\partial_{\mu} L(\varphi, X)=0 \longrightarrow L(\varphi, X)=-2 M=$ const,

where $M$ is arbitrary integration constant ${ }^{4}$ (the factor 2 is for later convenience).

\footnotetext{
${ }^{3}$ In the original papers [8-11] an alternative parametrization of $B_{\mu \nu \lambda}$ through 4 auxiliary scalar fields $\left\{\phi^{I}\right\}_{I=1, \ldots, 4}$ was used $-B_{\mu \nu \lambda}=\frac{1}{4} \varepsilon_{I J K L} \phi^{I} \partial_{\mu} \phi^{J} \partial_{\nu} \phi^{K} \partial_{\lambda} \phi^{L}$, so that $\Phi(B)=$ $\frac{1}{4 !} \varepsilon^{\mu \nu \kappa \lambda} \varepsilon_{I J K L} \partial_{\mu} \phi^{I} \partial_{\nu} \phi^{J} \partial_{\kappa} \phi^{K} \partial_{\lambda} \phi^{L}=\operatorname{det}\left\|\frac{\partial \phi^{I}}{\partial x^{\mu}}\right\|$. In a recent study [41] of general relativity as an extended canonical gauge theory a similar Jacobian representation of the covariant integration measure has appeared in terms of additional scalar fields. However, unlike the present case in the construction of Ref. [41] the additional scalar fields enter also in the proper Lagrangian.

${ }^{4}$ Dynamical constraints like the one on the scalar field Lagrangian in Eq. (7), which routinely appear in all instances of applying the nonRiemannian volume-form method in gravity-matter theories, resemble at first sight analogous constraints on scalar field Lagrangians in "Lagrangian multiplier gravity" [46]. We would like to point out that this formalism is in fact a special particular case of the more general approach based on non-Riemannian spacetime volume forms, which appeared around a decade earlier [8-11]. Dynamical constraints in the
} 
Already at this point it is important to stress that the scalar field dynamics is determined entirely by the firstorder differential equation - the dynamical constraint Eq. (7) $(X-V(\varphi)=-2 M$ in the simplest case of Eq. (3)). The standard second order differential equation Eq. (6) is in fact a consequence of Eq. (7) together with the energy-momentum conservation $\nabla^{\mu} T_{\mu \nu}=0$.

The physical meaning of the "measure" gauge field $B_{\mu \nu \lambda}$ (2) as well as the meaning of the integration constant $M$ are most straightforwardly seen within the canonical Hamiltonian treatment of (the scalar field part of) Eq. (1). Namely, upon introducing the short-hand notation

$$
\begin{aligned}
& \Phi(B)=\partial_{\mu} \mathcal{B}^{\mu}=\dot{\mathcal{B}}+\partial_{i} \mathcal{B}^{i}, \\
& \mathcal{B} \equiv \mathcal{B}^{0}=\frac{1}{3 !} \varepsilon^{m k l} B_{m k l}, \quad \mathcal{B}^{i} \equiv-\frac{1}{2} \varepsilon^{i k l} B_{0 k l},
\end{aligned}
$$

we have for the canonically conjugated momenta $\pi_{\mathcal{B}}, \pi_{\mathcal{B}^{i}}$, and $p_{\varphi}$ w.r.t. $\mathcal{B}, \mathcal{B}^{i}$, and $\varphi$ :

$\pi_{\mathcal{B}^{i}}=0, \quad \pi_{\mathcal{B}}=L(\varphi, X)$,

$p_{\varphi}=\left(\dot{\mathcal{B}}+\partial_{i} \mathcal{B}^{i}+\sqrt{-g}\right) \frac{\partial L}{\partial \dot{\varphi}}$.

The first relations in Eq. (9) represent primary Dirac firstclass constraints and, therefore, their canonically conjugate coordinates $\mathcal{B}^{i}$ ("electric" components of the auxiliary "measure" gauge field $B_{\mu \nu \lambda}$, cf. Eq. (8)) are pure-gauge degrees of freedom - in fact they are Lagrange multipliers for secondary Dirac first-class constraints (see Eq. (14) below). From the second relation in (9) we obtain the velocity $\dot{\varphi}=\dot{\varphi}\left(\varphi, \pi_{\mathcal{B}}\right)$ as a function of the canonical variables (in the simplest case of Eq. (3) $L(\varphi, X)=X-V(\varphi))$ :

$\dot{\varphi}=N^{i} \partial_{i} \varphi+N \sqrt{h^{i j} \partial_{i} \varphi \partial_{j} \varphi+2\left(V(\varphi)+\pi_{\mathcal{B}}\right)}$,

where we have used the standard ADM parametrization for the Riemannian metric:

$\mathrm{d} s^{2}=-N^{2} \mathrm{~d} t^{2}+h_{i j}\left(\mathrm{~d} x^{i}+N^{i} \mathrm{~d} t\right)\left(\mathrm{d} x^{j}+N^{j} \mathrm{~d} t\right)$.

Finally, from the last relation in Eq. (9) we obtain the velocity $\dot{\mathcal{B}}$ as a function of the canonical variables. Thus, inserting Eq. (10) and the second relation Eq. (9) in the expression for the canonical scalar field Hamiltonian:

$\mathcal{H}_{m}=p_{\varphi} \dot{\varphi}+\pi_{\mathcal{B}} \dot{\mathcal{B}}-\left(\dot{\mathcal{B}}+\partial_{i} \mathcal{B}^{i}+\sqrt{-g}\right) L(\varphi, X)$,

Footnote 4 continued

latter approach result from the equations of motion of the auxiliary "measure" gauge fields and, thus, they always involve arbitrary integration constants like $M$ in Eq. (7), as opposed to picking some a priori fixed constant within the "Lagrange multiplier gravity" formalism. For further advantages of the non-Riemannian volume-form formalism, see the final remarks in the Sect. 1. we arrive at the result

$$
\begin{aligned}
& \mathcal{H}_{m}=N^{i}\left(\partial_{i} \varphi p_{\varphi}\right) \\
& +N\left[p_{\varphi} \sqrt{h^{i j} \partial_{i} \varphi \partial_{j} \varphi+2\left(V(\varphi)+\pi_{\mathcal{B}}\right)}-\sqrt{h} \pi_{\mathcal{B}}\right] \\
& -\partial_{i} \mathcal{B}^{i} \pi_{\mathcal{B}} .
\end{aligned}
$$

i.e., a scalar field canonical Hamiltonian being linear combination of first-class constraints only.

The last term in Eq. (13) shows that the $\mathcal{B}^{i}$ are canonical Lagrange multipliers for the secondary Dirac first-class constraints:

$\partial_{i} \pi_{\mathcal{B}}=0 \longrightarrow \pi_{\mathcal{B}}=$ const $\equiv-2 M$.

The latter implies that also $\mathcal{B}$ (the "magnetic" component of the auxiliary "measure" gauge field $B_{\mu \nu \lambda}$, cf. Eq. (8)) is a pure-gauge degree of freedom. Clearly, Eq. (14) are the canonical Hamiltonian analog of Eq. (7) within the Lagrangian formalism. Therefore, the meaning of the arbitrary integration constant $2 M$ is the minus value of conserved Dirac-constrained canonical momentum conjugated to the "pure-gauge" magnetic component of the "measure" gauge field $B_{\mu \nu \lambda}$. Moreover, the second term in Eq. (13) shows that $M$ plays the role of a dynamically generated cosmological constant.

Adding the well-known canonical Hamiltonian of the Hilbert-Einstein action (up to a total derivative term [47]) the total canonical Hamiltonian of the gravity-scalar-field model Eq. (1) is the following linear combination of the first-class constraints:

$$
\begin{aligned}
\mathcal{H}_{\text {total }} & =N^{i} \mathcal{H}_{i}+N \mathcal{H}_{0}-\partial_{i} \mathcal{B}^{i} \pi_{\mathcal{B}} \\
\mathcal{H}_{i} \equiv & -2 D_{j} \pi_{i}^{j}+\partial_{i} \varphi p_{\varphi}, \\
\mathcal{H}_{0} \equiv & \frac{1}{\sqrt{h}}\left(\pi_{i j} \pi^{i j}-\frac{1}{2}\left(\pi_{i}^{i}\right)^{2}-\sqrt{h} R^{(3)}(h)\right. \\
& +p_{\varphi} \sqrt{h^{i j} \partial_{i} \varphi \partial_{j} \varphi+2\left(V(\varphi)+\pi_{\mathcal{B}}\right)}-\pi_{\mathcal{B}} \sqrt{h} .
\end{aligned}
$$

Here $\pi^{i j}$ denote canonically conjugated momenta of the spatial three-dimensional ADM metric $g_{i j}=h_{i j}, \sqrt{h}=$ $\sqrt{\operatorname{det}\left\|h_{i j}\right\|}, D_{i}$, and $R^{(3)}(h)$ denote covariant derivative and scalar curvature w.r.t. $h_{i j}$, respectively.

For more details as regards the canonical Hamiltonian treatment of gravity-matter theories with non-Riemannian volume forms we refer to $[27,28]$.

\section{Hidden symmetry, conservation laws, and "dust" fluid interpretation}

We go back to the Lagrangian formalism and consider Eq. (7). Multiplying its differential form $\partial_{\mu} L(\varphi, X) \equiv \partial_{\mu} \varphi \frac{\partial L}{\partial \varphi}+$ $\partial_{\mu} X \frac{\partial L}{\partial X}=0$ by the factor $-\frac{1}{2} g^{\mu \nu} \partial_{\nu} \varphi$ we get the following 
equivalent form of the dynamical Lagrangian constraint (7):

$\frac{\partial L}{\partial \varphi}-\frac{\partial_{\mu} \sqrt{X}}{\sqrt{X}} g^{\mu \nu} \partial_{\nu} \varphi \frac{\partial L}{\partial X}=0$.

Inserting $\frac{\partial L}{\partial \varphi}$ from Eq. (18) into $\varphi$-equations of motion Eq. (6) we immediately rewrite the latter in the following currentconservation law form (for later convenience we multiplied both sides by the numerical factor $\sqrt{2}$ ):

$\partial_{\mu}\left[(\Phi(B)+\sqrt{-g}) \sqrt{2 X} g^{\mu \nu} \partial_{\nu} \varphi \frac{\partial L}{\partial X}\right]=0$

or, equivalently, in a covariant form,

$\nabla_{\mu} J^{\mu}=0, \quad J^{\mu} \equiv\left(1+\frac{\Phi(B)}{\sqrt{-g}}\right) \sqrt{2 X} g^{\mu \nu} \partial_{\nu} \varphi \frac{\partial L}{\partial X}$.

In fact, we find a hidden (strongly nonlinear) Noether symmetry of the original action Eq. (1) which produces $J^{\mu}$, Eq. (20), as a genuine Noether conserved current. Indeed, the action Eq. (1) is invariant (modulo total derivative) under the following nonlinear symmetry transformations:

$$
\begin{aligned}
\delta_{\epsilon} \varphi & =\epsilon \sqrt{X}, \quad \delta_{\epsilon} g_{\mu \nu}=0, \\
\delta_{\epsilon} \mathcal{B}^{\mu} & =-\epsilon \frac{1}{2 \sqrt{X}} g^{\mu \nu} \partial_{\nu} \varphi(\Phi(B)+\sqrt{-g}),
\end{aligned}
$$

where the short-hand notation of Eq. (8) is used. Under Eq. (21) the action Eq. (1) transforms as $\delta_{\epsilon} S=\int \mathrm{d}^{4} x \partial_{\mu}(L(\varphi, X)$ $\left.\delta_{\epsilon} \mathcal{B}^{\mu}\right)$. Then the standard Noether procedure yields precisely $J^{\mu}$ Eq. (20) as the pertinent conserved current.

Let us particularly stress that the existence of the hidden symmetry of Eq. (21) of the action Eq. (1) does not depend on the specific form of the scalar field Lagrangian (3). The only requirement is that the kinetic term $X$ must be positive.

We can now rewrite $T_{\mu \nu}$ Eq. (5) and $J^{\mu}$ (20) in the following relativistic hydrodynamical form [taking into account Eq. (7)]:

$T_{\mu \nu}=\rho_{0} u_{\mu} u_{\nu}-2 M g_{\mu \nu}, \quad J^{\mu}=\rho_{0} u^{\mu}$,

where the integration constant $M$ appears as a dynamically generated cosmological constant and

$\rho_{0} \equiv\left(1+\frac{\Phi(B)}{\sqrt{-g}}\right) 2 X \frac{\partial L}{\partial X}$,

$u_{\mu} \equiv \frac{\partial_{\mu} \varphi}{\sqrt{2 X}} \quad\left(\right.$ note $\left.u^{\mu} u_{\mu}=-1\right)$.

Comparing Eq. (22) with the standard expression for a perfect fluid stress-energy tensor $T_{\mu \nu}=(\rho+p) u_{\mu} u_{\nu}+p g_{\mu \nu}$, we see that

$p=-2 M, \quad \rho=\rho_{0}+2 M \quad$ with $\rho_{0}$ as in (23),

i.e, the fluid tension is constant and negative, whereas $\rho_{0}$ in Eq. (23) and $2 M$ are the rest-mass and internal fluid energy densities, respectively (for general definitions, see i.e. [49]).
The energy-momentum tensor Eq. (22) consists of two parts with the following interpretation according to the standard $\Lambda$-CDM model [50-52] [using the notation $p=p_{\mathrm{DM}}+$ $p_{\mathrm{DE}}$ and $\rho=\rho_{\mathrm{DM}}+\rho_{\mathrm{DE}}$ in Eq. (24)]:

- A dark energy part given by the second cosmological constant term in $T_{\mu \nu}$ Eq. (22), which arises due to the dynamical constraint on the scalar field Lagrangian (7), or equivalently, by Eq. (24) with $p_{\mathrm{DE}}=-2 M, \rho_{\mathrm{DE}}=$ $2 M$.

- A dark matter part given by the first term in Eq. (22), or equivalently, by Eq. (24) with $p_{\mathrm{DM}}=0, \rho_{\mathrm{DM}}=\rho_{0}\left[\rho_{0}\right.$ as in Eq. (23)], which in fact describes a dust.

Indeed, the covariant conservation laws for the energymomentum tensor Eq. (22), $\nabla^{\mu} T_{\mu \nu}=0$, and the $J$ current Eq. (20) acquire the form

$\nabla^{\mu}\left(\rho_{0} u_{\mu} u_{v}\right)=0, \quad \nabla^{\mu}\left(\rho_{0} u_{\mu}\right)=0$,

both of which implying the geodesic equation for the "dust fluid" 4-velocity $u_{\nu}$ :

$u_{\mu} \nabla^{\mu} u_{v}=0$.

To conclude this section let us point out that the hidden symmetry transformation of the scalar field [first equation of Eq. (21)] can be equivalently represented as a specific fielddependent coordinate shift of the $\varphi$-field [taking into account the definition of $X$ in Eq. (3)]:

$$
\begin{aligned}
\delta_{\epsilon} \varphi(x) & =\epsilon \sqrt{X}=\varphi\left(x+\epsilon \zeta_{\varphi}(x)\right)-\varphi(x) \\
& =\epsilon \zeta_{\varphi}^{\mu}(x) \partial_{\mu} \varphi(x), \quad \zeta_{\varphi}^{\mu}=-\frac{1}{\sqrt{2}} u^{\mu} .
\end{aligned}
$$

Accordingly, the dust 4-velocity transforms under the hidden symmetry Eq. (21) or Eq. (27) as

$\delta_{\epsilon} u^{\mu}=\epsilon\left(g^{\mu \nu}+u^{\mu} u^{\nu}\right) \frac{\partial_{\nu} \sqrt{X}}{\sqrt{2 X}}$.

\section{Implications for cosmology}

Let us now consider the modified gravity-scalar-field model (1) with the hidden symmetry Eq. (21) describing simultaneously dark matter and dark energy in the context of cosmology. To this end let us take the Friedmann-LemaitreRobertson-Walker (FLRW) metric (see e.g. [48]):

$$
\mathrm{d} s^{2}=-\mathrm{d} t^{2}+a^{2}(t)\left[\frac{\mathrm{d} r^{2}}{1-K r^{2}}+r^{2}\left(\mathrm{~d} \theta^{2}+\sin ^{2} \theta \mathrm{d} \phi^{2}\right)\right],
$$


and consider the associated Friedmann equations:

$\frac{\ddot{a}}{a}=-\frac{1}{12}(\rho+3 p), \quad H^{2}+\frac{K}{a^{2}}=\frac{1}{6} \rho, \quad H \equiv \frac{\dot{a}}{a}$,

describing the universe's evolution. In the present case we have for the pressure $p$ and the full energy density $\rho$ the explicit expressions Eq. (24). Also now $\varphi=\varphi(t)$, so that $X=\frac{1}{2} \dot{\varphi}^{2}$ and $u_{\mu}=(1,0,0,0)$. The $J^{\mu}$ current conservation Eq. (25) now reads

$\nabla^{\mu}\left(\rho_{0} u_{\mu}\right)=0 \rightarrow \frac{\mathrm{d}}{\mathrm{d} t}\left(a^{3} \rho_{0}\right)=0 \rightarrow \rho_{0}=\frac{c_{0}}{a^{3}}$,

where the last relation is the typical cosmological dust solution (see e.g. [51]) with $c_{0}=$ const. Inserting in Eq. (31) the explicit expression Eq. (23) for $\rho_{0}$ we obtain a solution for the non-Riemannian integration measure density $\Phi(B)=c_{0}\left(2 X \frac{\partial L}{\partial X}\right)^{-1}-a^{3}$, or in the simplest case for the scalar Lagrangian $\left(L=\frac{1}{2} \dot{\varphi}^{2}-V(\varphi)\right)$

$\Phi(B)=\frac{c_{0}}{\dot{\varphi}^{2}}-a^{3}$.

Let us particularly stress that the solution Eq. (31) for the dust (dark matter) energy density $\rho_{0}$ [last relation in (24)] does not depend on the specific form of the scalar Lagrangian (cf. Eq. (3)) and the details of the dynamics of $\varphi(t)$ :

$L(\varphi, X)=\frac{A_{1}(\varphi)}{2} \dot{\varphi}^{2}+\frac{A_{2}(\varphi)}{4} \dot{\varphi}^{4}+\cdots-V(\varphi)$.

Taking into account Eqs. (24) and (31), the Friedmann equations (30) acquire the form

$\frac{\ddot{a}}{a}=-\frac{1}{12}\left(\frac{c_{0}}{a^{3}}-4 M\right), \quad \frac{\dot{a}^{2}}{a^{2}}+\frac{k}{a^{2}}=\frac{1}{6}\left(\frac{c_{0}}{a^{3}}+2 M\right)$,

and, thus, the solution for $a=a(t)$ does not depend either on the specific form of the scalar Lagrangian Eq. (33) and the details of the dynamics of $\varphi(t)$. An exact solution for $a(t)$ of the second equation of Eq. (34) when $k=0$ was given in Ref. [50]. In the general case, including radiation, exact solutions for $a(t)$ in terms of elliptic functions can be found in Ref. [53].

In fact, concerning the cosmological solutions of Eqs. (31) and (34), the only requirement for $L(\varphi, X)(33)$ comes from the dynamical constraint of Eq. (7) on Eq. (33):

$L(\varphi, X)=-2 M \rightarrow V(\varphi)>2 M$.

In general the inequality $V(\varphi)>2 M$ might define classical forbidden regions for $\varphi(t)$ (where $V(\varphi)<2 M$ ), including turning points $\varphi_{0}$ [where $V\left(\varphi_{0}\right)=2 M$ ]. In view of later applications (see the discussion of obtaining growing dark energy below) we will require

$V(\varphi)>2 M$ for all $\varphi$,

so that we will have a purely monotonic behavior for $\varphi(t)$ (cf. Eq. (38) below).
The dynamics of the scalar field $\varphi(t)$ itself is given by the first-order differential equation (35). Although it does not affect the cosmological solutions, nevertheless, it is worth mentioning the following property. Taking time derivative on both sides of Eq. (35) we obtain second order evolution equation for $\varphi(t)$ :

$\ddot{\varphi}\left(A_{1}(\varphi)+\frac{1}{2} A_{2}(\varphi) \dot{\varphi}^{2}+\cdots\right)$
$\quad+\frac{1}{2} A_{1}^{\prime}(\varphi) \dot{\varphi}^{2}+\frac{1}{4} A_{2}^{\prime}(\varphi) \dot{\varphi}^{4}+\cdots-\frac{\partial V}{\partial \varphi}=0$.

In particular, for the standard scalar Lagrangian $L=\frac{1}{2} \dot{\varphi}^{2}-$ $V(\varphi)$ Eqs. (35) and (37) read, accordingly,

$\dot{\varphi}^{2}=2(V(\varphi)-2 M)$

$\rightarrow \int_{\varphi(0)}^{\varphi(t)} \frac{d \varphi}{\sqrt{2(V(\varphi)-2 M)}}= \pm t$

$\ddot{\varphi}-\frac{\partial V}{\partial \varphi}=0$,

where we specifically stress on the opposite sign in the force term in the second order $\varphi$-equation of motion Eq. (39). Due to the dynamical constraint on $V(\varphi)$ in Eq. (36), choosing the + sign, the integral in Eq. (38) yields $\varphi(t)$ monotonically growing with $t$.

Let us now consider a perturbation of the initial modifiedmeasure gravity-scalar-field action Eq. (1) by some additional scalar potential $U(\varphi)$ independent of the initial potential $V(\varphi)$ :

$$
\begin{aligned}
\widetilde{S}= & \int \mathrm{d}^{4} x \sqrt{-g} R+\int \mathrm{d}^{4} x(\sqrt{-g}+\Phi(B)) L(\varphi, X) \\
& -\int \mathrm{d}^{4} x \sqrt{-g} U(\varphi) .
\end{aligned}
$$

An important property of the perturbed action Eq. (40) is that once again the scalar field $\varphi$-dynamics is given by the unperturbed dynamical constraint Eq. (7), in particular, by Eq. (37) or Eq. (38) in the case of FLRW metric Eq. (29). Let us strongly emphasize that the latter are completely independent of the perturbing scalar potential $U(\varphi)$.

The associated scalar field energy-momentum tensor now reads (cf. Eqs. (22) and (24))

$$
\begin{aligned}
\widetilde{T}_{\mu \nu} & =\rho_{0} u_{\mu} u_{\nu}+g_{\mu \nu}(-2 M-U(\varphi)) \\
& \equiv(\widetilde{\rho}+\widetilde{p}) u_{\mu} u_{\nu}+\widetilde{p} g_{\mu \nu}, \\
\widetilde{\rho} & =\rho_{0}+2 M+U, \quad \widetilde{p}=-2 M-U,
\end{aligned}
$$

where again the notation of Eq. (23) is used.

The perturbed energy-momentum Eq. (41) conservation $\nabla^{\mu} \widetilde{T}_{\mu \nu}=0$ now implies (cf. Eqs. (25) and (26))

$\nabla^{\mu}\left(\rho_{0} u_{\mu}\right)-\sqrt{2 X} \frac{\partial U}{\partial \varphi}=0, \quad u_{\mu} \nabla^{\mu} u_{v}=0$. 
While we again obtain the geodesic equation for the dark matter "fluid" 4-velocity, in the perturbed case the action Eq. (40) does not any more possess the hidden symmetry Eq. (21) and, therefore, the conservation of the Noether current $J^{\mu}=\rho_{0} u^{\mu}$ Eq. (22) is now replaced by the first equation of Eq. (43). In the case of the FLRW metric, Eq. (29), the latter acquires the well-known form

$\frac{\mathrm{d}}{\mathrm{d} t}\left(a^{3} \widetilde{\rho}\right)+\widetilde{p} \frac{\mathrm{d}}{\mathrm{d} t} a^{3}=0$,

where the notation of Eq. (42) for the total perturbed energy density and pressure is used.

As already stressed above, the dynamics of the scalar field does not depend at all on the presence of the perturbing scalar potential $U(\varphi)$. Therefore, if we choose the perturbation $U(\varphi)$ in (40) to be a growing function at large $\varphi$ [e.g., $U(\varphi) \sim e^{\alpha \varphi}, \alpha$ small positive], then, when $\varphi(t)$ evolves through Eq. (38) to large positive values, it (slowly) "climbs" up the perturbing potential $U(\varphi)$ and according to the expression $2 M+U(\varphi)$ for the dark energy density (cf. Eq. (41)), the latter will (slowly) grow! Let us emphasize that in this way we obtain a growing dark energy of the "late" universe without any pathologies in the universe' evolution like "cosmic doomsday" or future singularities [38,39], see also [40].

Taking another example of perturbation in Eq. (40) of the type $U(\varphi) \sim \tanh (\alpha \varphi)$ for large $\varphi$, then after (slowly) growing of the dark energy density $2 M+U(\varphi)$ will asymptotically (for $t \rightarrow+\infty$ ) approach a finite constant value.

\section{Conclusions}

Let us recapitulate the main points above:

- Employing a non-Riemannian volume form (alternative covariant integration measure density independent of the Riemannian metric) in the modified-measure gravityscalar-field action Eq. (1) produces naturally a dynamically generated cosmological constant (identified as dark energy) in the form of an arbitrary integration constant in solving the equations of motion Eq. (7) corresponding to the auxiliary "measure" gauge fields.

- The modified-measure gravity-scalar-field action Eq. (1) possesses a hidden Noether symmetry Eq. (21) acting on the scalar field and the "measure" gauge fields (but leaving the Riemannian metric untouched), whose associated Noether conserved current Eq. (20) provides a relativistic hydrodynamical interpretation of the energy-momentum tensor Eq. (22) describing two decoupled matter components - a "dust" (dark matter) and a constant negative pressure (dark energy) ones.

- The above unified description of dark energy and dark matter is insensitive w.r.t. the specific form of the scalar field Lagrangian (which might be of higher order "kessence" type) and the details of the underlying dynamics of the scalar field.

- Upon appropriately perturbing the modified-measure gravity-scalar-field action Eq. (40), which breaks the above hidden symmetry, we find a way to obtain growing dark energy in the present universe's epoch without evolution pathologies.

Straightforward quantization (e.g., via a functional integral) of the scalar field action in Eq. (1), which is required to study possible quantum radiative instabilities within the cosmological constant problem, does not allow us the use the standard quantum field theoretic methods (standard perturbative expansion, Feynman diagrams and their renormalization). This is due to the essential nonlinearity (square root) in the expression for the corresponding scalar field canonical Hamiltonian Eq. (13) (even in flat spacetime $N=1, h_{i j}=$ $\delta_{i j}$ ) and, especially, because it is linear (instead of the usual quadratic) function of the conjugated canonical momentum $p_{\varphi}$.

Canonical Hamiltonian quantization of the full gravityscalar-field action Eq. (1) was studied in [54] in the reduced case of the FLRW cosmological metric Eq. (29) and purely time-dependent scalar field $\varphi$. Upon an appropriate change of variables the corresponding quantum Wheeler-DeWitt equation was reduced (in the case of zero FLRW spatial curvature) to the Schrödinger equation for the inverted harmonic oscillator.

Acknowledgments We gratefully acknowledge support of our collaboration through the academic exchange agreement between the BenGurion University in Beer-Sheva, Israel, and the Bulgarian Academy of Sciences. S.P. and E.N. have received partial support from European COST actions MP-1210 and MP-1405, respectively, as well from Bulgarian National Science Fund Grant DFNI-T02/6. E.G. thanks Samuel Lepe for discussions. We are also grateful to Ali Chamseddine, the referees and the editor for useful remarks.

Open Access This article is distributed under the terms of the Creative Commons Attribution 4.0 International License (http://creativecomm ons.org/licenses/by/4.0/), which permits unrestricted use, distribution, and reproduction in any medium, provided you give appropriate credit to the original author(s) and the source, provide a link to the Creative Commons license, and indicate if changes were made.

Funded by SCOAP ${ }^{3}$.

\section{References}

1. A.G. Riess et al., Astron. J. 116, 1009-1038 (1998). arXiv:astro-ph/9805201

2. S. Perlmutter et al., Astrophys. J. 517, 565-586 (1999). arXiv:astro-ph/9812133

3. A.G. Riess et al., Astrophys. J. 607, 665-687 (2004). arXiv:astro-ph/0402512 
4. K. Garrett, G. Duda, Adv. Astron. 2011, 968283 (2011). arXiv: 1006.2483

5. V. Sahni, A.A. Starobinsky, Int. J. Mod. Phys. D 9, 373 (2000). arXiv:astro-ph/9904398

6. P.J.E. Peebles, B. Ratra, Rev. Mod. Phys. 75, 559 (2003). arXiv:astro-ph/0207347

7. V. Sahni, Lect. Notes Phys. 653, 141 (2004). arXiv:astro-ph/0403324

8. E.I. Guendelman, Mod. Phys. Lett. A 14, 1043-1052 (1999). arXiv:gr-qc/9901017

9. E. Guendelman, A. Kaganovich, Phys. Rev. D 60, 065004 (1999). arXiv:gr-qc/9905029

10. E.I. Guendelman, Found. Phys. 31, 1019-1037 (2001). arXiv:hep-th/0011049

11. E. Guendelman, O. Katz, Class. Q. Grav. 20, 1715-1728 (2003). arXiv:gr-qc/0211095

12. E. Guendelman, D. Singleton, N. Yongram, JCAP 1211, 044 (2012). arXiv:1205.1056

13. S. Ansoldi, E. Guendelman, JCAP 1305, $036 \quad$ (2013). arXiv: 1209.4758

14. A. Aviles, N. Cruz, J. Klapp, O. Luongo, Gen. Rel. Grav. 47, 63 (2015). arXiv:1412.4185

15. A.Yu. Kamenshchik, U. Moschella, V. Pasquier, Phys. Lett. B 511, 265-268 (2001). arXiv:gr-qc/0103004

16. N. Bilic, G. Tupper, R. Viollier, Phys. Lett. B 535, 17-21 (2002). arXiv:astro-ph/0111325

17. N. Bilic, G. Tupper, R. Viollier, Phys. Rev. D 80, 023515 (2009). arXiv:0809.0375

18. N. Bilic, G. Tupper, R. Viollier, J. Phys. A 40, 6877 (2007). arXiv:gr-qc/0610104

19. A. Chamseddine, V. Mukhanov, JHEP 1311, 135 (2013). arXiv: 1308.5410

20. A. Chamseddine, V. Mukhanov, A. Vikman, JCAP 1406, 017 (2014). arXiv:1403.3961

21. M. Chaichian, J. Kluson, M. Oksanen, A. Tureanu, JHEP 1412, 102 (2014). arXiv: 1404.4008

22. A. Pourtsidou, C. Skordis, E.J. Copeland, Phys. Rev. D 88, 083505 (2013). arXiv:1307.0458

23. E. Guendelman, Class. Q. Grav. 17, 3673-3680 (2000). arXiv:hep-th/0005041

24. E. Guendelman, A. Kaganovich, E. Nissimov, S. Pacheva, Phys. Rev. D 66, 046003 (2002). arXiv:hep-th/0203024

25. E. Guendelman, P. Labrana, Int. J. Mod. Phys. D 22, 1330018 (2013). arXiv:1303.7267

26. E. Guendelman, H. Nishino, S. Rajpoot, Phys. Lett. B 732, 156 (2014). arXiv: 1403.4199

27. E. Guendelman, E. Nissimov, S. Pacheva, in Eight Mathematical Physics Meeting, ed. by B. Dragovic, I. Salom (Belgrade Inst. Phys. Press, Belgrade, 2015), pp. 93-103. arXiv:1407.6281v4

28. E. Guendelman, E. Nissimov, S. Pacheva, Int. J. Mod. Phys. 30, 1550133 (2015). arXiv: 1504.01031

29. M. Henneaux, C. Teitelboim, Phys. Lett. 222B, 195-199 (1989)
30. A. Einstein, Ann. Phys. 49, 769 (1916) [translated and included in The Principle of Relativity, by H.A. Lorentz et al. (Dover Press, New York, 1923)]

31. A. Einstein, Sitzungsber. Preuss. Akad. Wiss. Berlin (Math. Phys.) 1919, 433 (1919) [translated and included in The Principle of Relativity, ed. by H.A. Lorentz et al. (Dover Press, New York, 1923)]

32. J. van der Bij, H. van Dam, Y.J. Ng, Physica A 116, 307-320 (1982)

33. A. Zee, in High Energy Physics, ed. by S.L. Mintz, A. Perlmutter. Proceedings of the 20th Annual Orbis Scientiae (Plennum Press, New York, 1985)

34. R. Bufalo, M. Oksanen, A. Tureanu. arXiv:1505.04978

35. E. Guendelman, R. Herrera, P. Labrana, E. Nissimov, S. Pacheva, Gen. Rel. Grav. 47, 10 (2015). arXiv:1408.5344v4

36. E. Guendelman, E. Nissimov, S. Pacheva, M. Vasihoun, Bulg. J. Phys. 41, 123-129 (2014). arXiv:1404.4733

37. E. Guendelman, E. Nissimov, S. Pacheva, M. Vasihoun, in Eight Mathematical Physics Meeting, ed. by B. Dragovic, I. Salom (Belgrade Inst. Phys. Press, Belgrade, 2015), pp. 105-115. arXiv: 1501.05518

38. R.R. Caldwell, M. Kamionkowski, N.N. Weinberg, Phys. Rev. Lett. 91, 071301 (2003). arXiv:astro-ph/0302506

39. J.D. Barrow, Class. Q. Grav. 21, L79-L82 (2004). arXiv:gr-qc/0403084

40. A.V. Astashenok, S. Nojiri, S.D. Odintsov, A.V. Yurov, Phys. Lett. B 709, 396-403 (2012). arXiv:1201.4056

41. J. Struckmeier, Phys. Rev. D 91, 085030 (2015). arXiv:1411.1558 [gr-qc]

42. T. Chiba, T. Okabe, M. Yamaguchi, Phys. Rev. D 62, 023511 (2000). arXiv:astro-ph/9912463

43. C. Armendariz-Picon, V. Mukhanov, P. Steinhardt, Phys. Rev. Lett. 85, 4438 (2000). arXiv:astro-ph/0004134

44. C. Armendariz-Picon, V. Mukhanov, P. Steinhardt, Phys. Rev. D 63, 103510 (2001). arXiv:astro-ph/0006373

45. T. Chiba, Phys. Rev. D 66, 063514 (2002). arXiv:astro-ph/0206298

46. E. Lim, I. Sawicki, A. Vikman, JCAP 1005, 012 (2010). arXiv: 1003.5751

47. T. Regge, C. Teitelboim, Ann. Phys. 88, 286-318 (1974)

48. S. Weinberg, Gravitation and Cosmology-Principles and Applications of the General Theory of Relativity (Wiley, New York, 1972)

49. L. Rezzolla, O. Zanotti, Relativistic Hydrodynamics (Oxford University Press, Oxford, 2013)

50. J. Frieman, M. Turner, D. Huterer, Ann. Rev. Astron. Astrophys. 46, 385-432 (2008). arXiv:0803.0982

51. A. Liddle, Introduction to Modern Cosmology, 2nd edn. (Wiley, Chichester, 2003)

52. S. Dodelson, Modern Cosmology (Academic Press, San Diego, 2003)

53. L.I. Kharbediya, Sov. Astron. 27, 380-384 (1983) [Engl. transl. Astronom. Zhurnal 60, 648-655 (1983)]

54. E. Guendelman, E. Nissimov, S. Pacheva. arXiv:1509.01512 